\title{
Correction to: community-acquired pneumonia in children - a changing spectrum of disease
}

David M. le Roux ${ }^{1,2}$ • Heather J. Zar ${ }^{1}$

Published online: 6 November 2017

(C) Springer-Verlag GmbH Germany 2017

Correction to: Pediatr Radiol (2017) 47:1392-1398.

https://doi.org/10.1007/s00247-017-3827-8

The published version of this article unfortunately contained an error. Corresponding author information included only the mailing address but not the affiliations. Full corresponding author information is given below. The publisher apologizes.

The online version of the original article can be found at https://doi.org/ $10.1007 / \mathrm{s} 00247-017-3827-8$

David M. le Roux

Dave.leRoux@uct.ac.za

1 Department of Paediatrics and Child Health, Red Cross War Memorial Children's Hospital and MRC Unit on Child and Adolescent Health, University of Cape Town, 5th Floor ICH Building Klipfontein Road, Cape Town 7700, South Africa

2 Department of Paediatrics, New Somerset Hospital, Cape Town, South Africa 